\title{
Smoking and renal function in people living with human immunodeficiency virus: a Danish nationwide cohort study
}

This article was published in the following Dove Press journal:

Clinical Epidemiology

28 August 2015

Number of times this article has been viewed

\author{
Magnus Glindvad Ahlström' \\ Bo Feldt-Rasmussen ${ }^{2}$ \\ Rebecca Legarth' \\ Gitte Kronborg ${ }^{3}$ \\ Court Pedersen ${ }^{4}$ \\ Carsten Schade Larsen ${ }^{5}$ \\ Jan Gerstoft ${ }^{\prime}$ \\ Niels Obel' \\ 'Department of Infectious Diseases, \\ 2Department of Nephrology, \\ Copenhagen University Hospital, \\ Rigshospitalet, Copenhagen, \\ ${ }^{3}$ Department of Infectious Diseases, \\ Copenhagen University Hospital, \\ Hvidovre, ${ }^{4}$ Department of Infectious \\ Diseases, Odense University Hospital, \\ Odense, ${ }^{5}$ Department of Infectious \\ Diseases, Aarhus University Hospital, \\ Skejby, Aarhus, Denmark
}

Introduction: Smoking is a main risk factor for morbidity and mortality in people living with human immunodeficiency virus (PLHIV), but its potential association with renal impairment remains to be established.

Methods: We did a nationwide population-based cohort study in Danish PLHIV to evaluate the association between smoking status and 1) overall renal function and risk of chronic kidney disease (CKD), 2) risk of any renal replacement therapy (aRRT), and 3) mortality following aRRT. We calculated estimated creatinine clearance using the Cockcroft-Gault equation (CG$\mathrm{CrCl}$ ), and evaluated renal function graphically. We calculated cumulative incidence of CKD (defined as two consecutive CG-CrCls of $\leq 60 \mathrm{~mL} / \mathrm{min}, \geq 3$ months apart) and aRRT and used Cox regression models to calculate incidence rate ratios (IRRs) for risk of CKD, aRRT, and mortality rate ratios (MRRs) following aRRT.

Results: From the Danish HIV Cohort Study, we identified 1,475 never smokers, 768 previous smokers, and 2,272 current smokers. During study period, we observed no association of smoking status with overall renal function. Previous and current smoking was not associated with increased risk of CKD (adjusted IRR: 1.1, 95\% confidence interval [CI]: 0.7-1.7; adjusted IRR: 1.3, 95\% CI: 0.9-1.8) or aRRT (adjusted IRR: 0.8, 95\% CI: 0.4-1.7; adjusted IRR: 0.9, 95\% CI: $0.5-1.7)$. Mortality following aRRT was high in PLHIV and increased in smokers vs never smokers (adjusted MRR: 3.8, 95\% CI: 1.3-11.2).

Conclusion: In Danish PLHIV, we observed no strong association between smoking status and renal function, risk of CKD, or risk of aRRT, but mortality was increased in smokers following aRRT.

Keywords: chronic kidney disease, renal replacement therapy, mortality, creatinine clearance, incidence rate ratio, mortality rate ratio

\section{Introduction}

Since the introduction of highly active antiretroviral therapy (HAART), the natural course of human immunodeficiency virus (HIV) infection has changed dramatically from being invariably fatal to a chronic disease with an overall favorable prognosis. However, people living with HIV (PLHIV) still suffer from a significantly increased all-cause mortality compared to the background population. ${ }^{1}$

PLHIV have a faster decline in renal function and a higher risk of any renal replacement therapy (aRRT) and end-stage renal disease (ESRD) compared to the background population, but the mechanisms of the deterioration in renal function are not fully understood. $^{2-4}$ Exposure to antiretrovirals (eg, tenofovir and atazanavir) has been associated with deterioration of renal function. ${ }^{5-7}$ Furthermore, HIV has been found to replicate in other cells than CD4 cells, for example, renal epithelial cells. ${ }^{8}$ This seems
Ahlström

Department of Infectious Diseases, Copenhagen University Hospital,

Rigshospitalet, Blegdamsvej 9,

2200 Copenhagen N, Denmark

Tel +4520632365

Fax +45 35456648

Email magnus.rasch@gmail.com submit your manuscript $\mid$ www.dovepress.com

Dovepress

http://dx.doi.org// 0.2147/CLEP.S83530 
to be directly associated with HIV-associated nephropathy seen mainly in PLHIV of black-African origin. ${ }^{9}$

Recent studies indicate that lifestyle-related factors (eg, smoking and other substance abuse) are more important than HIV-related factors as predictors of long-term survival among PLHIV. ${ }^{10-13}$

We hypothesized that smoking is a risk factor for decline in renal function, chronic kidney disease (CKD), aRRT, and death following aRRT in PLHIV. We therefore assessed the association between smoking status and 1) renal function and CKD, 2) risk of aRRT, and 3) mortality following aRRT.

\section{Methods \\ Study design}

We performed a population-based cohort study divided into three parts in which we estimated the association of smoking with the following: 1) overall renal function, evaluated by estimated creatinine clearance calculated with the Cockcroft-Gault equation (CG-CrCl), and the risk of CKD (as defined in the section Categorization of smoking, renal function, $\mathrm{CKD}$ and CD4 cell count), 2) risk of aRRT, and 3) mortality following aRRT.

\section{Setting}

Denmark had a population of approximately 5.5 million people, and an estimated HIV prevalence of $0.1 \%$ among adults in December 2013. ${ }^{14}$ PLHIV are treated in eight specialized HIV care centers and are seen on an outpatient basis at intended intervals of 12-24 weeks. Antiretroviral treatment is provided free of charge. Management of PLHIV is well organized, and rates of treatment failure and loss to follow-up are low. ${ }^{15,16}$

\section{Data sources - registries}

The unique ten-digit personal identification number assigned to all Danish residents at birth or immigration was used to track individuals in the following registries.

The Danish HIV Cohort Study (DHCS) is a prospective study of all PLHIV, 16 years or older at diagnosis, treated at Danish HIV centers after January 1, 1995. Individuals are consecutively enrolled, and data are annually updated and include demographics, date of HIV diagnosis, acquired immunodeficiency syndrome (AIDS)-defining events, and antiretroviral treatment. CD4 cell counts and viral loads are extracted electronically from laboratory data files. The study is described in detail elsewhere. ${ }^{17,18}$

Data on vital status, residency, and migration were extracted from the Danish Civil Registration System (DCRS) which was established in 1968 and stores information on all Danish residents. ${ }^{19}$
From the Danish National Hospital Registry (DNHR), we identified dialysis according to the International Classification of Diseases tenth revision (ICD-10) as BJFD.xx, ZZ43.40ZZ43.50 or International Classification of Diseases eighth revision (ICD-8) as 92390, 94399, 94340, and 94350. aRRT was defined as the first date of dialysis. With this definition of aRRT, we include both patients with chronic renal replacement therapy (cRRT) and patients who develop acute renal failure and require acute dialysis. We defined cRRT as dialysis for $\geq 3$ months. Furthermore, we identified individuals with diagnoses of hypertension (ICD-10: DI10DI15.9 and ICD-8: 40009-41499) and diagnoses of diabetes (ICD-10: DE10.0-DE14.9 and ICD-8: 24900-25009). DNHR is described in detail elsewhere. ${ }^{20}$

\section{Categorization of smoking, renal function, CKD, and CD4 cell count}

Data on smoking were obtained by interview. Individuals were categorized as smokers if they smoked any type of tobacco at least once a week or if they categorized themselves as smokers. Individuals were categorized as never, previous, and current smokers according to their status at study inclusion and did not change category during study period.

Serum creatinines were extracted from electronic laboratory databases and included all serum creatinines measured in the period 1995-2013 during inpatient admittances and outpatient planned controls. All serum creatinines were standardized according to the Jaffe method as described by the local laboratories. As an estimate of glomerular filtration rate (GFR), we calculated CG-CrCl without correction of body surface area. ${ }^{21,22}$

$$
\begin{aligned}
& \mathrm{CG}-\mathrm{CrCl}=(140-\text { age }) \times \text { weight }(\text { in } \mathrm{kg}) \\
& \times \frac{1.04}{\text { serum }- \text { creatinine }\left(\text { in } \frac{\mu \mathrm{mol}}{\mathrm{L}}\right)}(\text { if female }) \\
& \mathrm{CG}-\mathrm{CrCl}=(140-\text { age }) \times \text { weight }(\text { in } \mathrm{kg}) \\
& \left.\times \frac{1.23}{\text { serum }- \text { creatinine }\left(\text { in } \frac{\mu \mathrm{mol}}{\mathrm{L}}\right)} \text { (if male }\right)
\end{aligned}
$$

To calculate CG-CrCl, the age at the date of the actual serum creatinine measurement and the weight closest to that date were used. Thus, an individual with only one weight available was assumed to have the same weight throughout the entire study period.

In sensitivity analyses, we used the CKD epidemiological collaboration (CKD-EPI) equation to estimate GFR. ${ }^{23}$ 


$$
\begin{aligned}
& \mathrm{GFR}_{\text {CKD-EPI }}=141 \times \min \left(\frac{\text { serum }- \text { creatinine }}{\kappa}, 1\right)^{\alpha} \\
& \times \max \left(\frac{\text { serum }- \text { creatinine }}{\kappa}, 1\right)^{-1.209} \times 0.993^{\text {age }} \\
& \quad \times 1.018[\text { if female }] \times 1.159[\text { if black }]
\end{aligned}
$$

where serum creatinine is measured in $\mu \mathrm{mol} / \mathrm{L}, \kappa$ is 61.9 for females and 79.6 for males, $\alpha$ is -0.329 for females and -0.411 for males, min indicates the minimum of serum creatinine/ $\kappa$ or 1 , and max indicates the maximum of serum creatinine $/ \kappa$ or 1 . CKD was defined as two consecutive CG-CrCls of $\leq 60 \mathrm{~mL} / \mathrm{min}, \geq 3$ months apart. The date of the second $\mathrm{CG}-\mathrm{CrCl}$ measurement was defined as date of $\mathrm{CKD}$. $\mathrm{CD} 4$ cell count and $\mathrm{CG}-\mathrm{CrCl}$ at study inclusion were defined as the $\mathrm{CD} 4$ cell count and the $\mathrm{CG}-\mathrm{CrCl}$ closest to and within 2 years before and 6 months after study inclusion.

\section{Study population}

We included all PLHIV who 1) had available data on smoking status, 2) had a unique Danish person identification number, 3) were 16 years or older at study inclusion, 4) were alive and living in Denmark at study inclusion, and 5) did not have aRRT at or prior to study inclusion. This population was included in part 2.

In part 1, the following inclusion criteria were added. Individuals who 1) had at least three $\mathrm{CG}-\mathrm{CrCls}$ available during the study period, 2) had data on weight available, and 3) did not have CKD prior to study inclusion, for analyses evaluating risk of CKD.

In part 3, all patients from part 2 with aRRT were included.

\section{Study design}

\section{Part I - smoking, renal function, and CKD}

For the assessment of overall renal function over time, time was calculated from January 1, 1995, date of HIV diagnosis, date of first available data on smoking, first available CG$\mathrm{CrCl}$ or date of immigration whichever occurred last to date of aRRT, last available CG-CrCl, loss to follow-up, emigration, death, or 10 years after study inclusion, whichever occurred first. Observation time was divided into intervals of 3 months starting from study inclusion. If an individual had more than one $\mathrm{CG}-\mathrm{CrCl}$ within a 3-month interval, median $\mathrm{CG}-\mathrm{CrCl}$ was used. If no $\mathrm{CG}-\mathrm{CrCl}$ was recorded within a 3-month interval, we calculated the $\mathrm{CG}-\mathrm{CrCl}$ as a weighted mean of the two nearest $\mathrm{CG}-\mathrm{CrCl}$ measurements, thereby assuming linearity between the two measurements. In consequence, all individuals contributed with a $\mathrm{CG}-\mathrm{CrCl}$ in every 3-month time interval. We then graphically evaluated median $\mathrm{CG}-\mathrm{CrCl}$ according to smoking status for every 3-month interval. When we evaluated risk of CKD, time was calculated from January 1, 1995, date of HIV diagnosis, date of first available data on smoking, first available $\mathrm{CG}-\mathrm{CrCl}$ or date of immigration whichever occurred last to date of $\mathrm{CKD}$, last available $\mathrm{CG}-\mathrm{CrCl}$, loss to follow-up, emigration, death, or 10 years after study inclusion, whichever occurred first. Outcome was time to CKD. We calculated cumulative incidence with death, emigration, and loss to follow-up as competing risks to estimate cumulative risk of $\mathrm{CKD}$. We calculated incidence rates (IRs) and used Cox regression to calculate incidence rate ratios (IRRs) of CKD. The following covariates were included in the final model: gender (male vs female), race (Caucasian, African, or other), route of infection (men who have sex with men, intravenous drug use [IDU], heterosexually infected, or other), CD4 cell count ( $<200$ cells $/ \mu \mathrm{L}$ vs $\geq 200$ cells $/ \mu \mathrm{L})$ at study inclusion, and CG-CrCl ( $<90 \mathrm{~mL} / \mathrm{min}$ vs $\geq 90 \mathrm{~mL} / \mathrm{min}$ ) at study inclusion. The following covariates were included as time-updated variables: age (5-year intervals), HAART exposure (yes/no), tenofovir exposure (yes/no), diabetes (yes/no), hypertension (yes/no), and AIDS (yes/no). In sensitivity analyses, we estimated GFR with the CKD-EPI equation.

\section{Part 2 - smoking and aRRT}

Time was calculated from January 1, 1995, date of HIV diagnosis, date of first available data on smoking or date of immigration whichever occurred last to date of aRRT, loss to follow-up, emigration, death, or 10 years after study inclusion, whichever occurred first. Outcome was time to aRRT, as defined earlier.

We calculated cumulative incidence with death, emigration, and loss to follow-up as competing risks to estimate cumulative risk of aRRT. We calculated IRs and used Cox regression to calculate IRR of aRRT. The following covariates were included in the final model: gender (male vs female), race (Caucasian, African, or other), route of infection (men who have sex with men, IDU, heterosexually infected, or other $)$, and CD4 cell count $(<200$ cells $/ \mu \mathrm{L}$ vs $\geq 200$ cells $/ \mu \mathrm{L})$ at study inclusion. The following covariates were included as time-updated variables: age (5-year intervals), HAART exposure (yes/no), tenofovir exposure (yes/no), diabetes (yes/no), hypertension (yes/no), and AIDS (yes/no). To test for effect modification, we stratified the analyses on gender, route of infection, race, and CD4 cell count at study inclusion. We did sensitivity analyses excluding IDUs from the analyses and analyses excluding 
individuals of non-Danish origin. Furthermore, we analyzed the risk of cRRT according to smoking status.

\section{Part 3 - smoking and mortality following aRRT}

Time was calculated from aRRT to death, emigration, loss to follow-up, December 31, 2013, or 56 days after study inclusion whichever came first. Outcome was time from aRRT to death, and Cox regression analyses were used to estimate mortality rate ratios (MRRs). We included age at inclusion and gender as covariates in the model. We did not include comorbidities in the adjusted analyses as these covariates most likely are facilitators and not confounders of the increased mortality in smokers. Kaplan-Meier survival tables were used to construct survival curves following aRRT. In secondary analyses, IDUs were excluded from the analyses. Furthermore, we did a sensitivity analyses only including individuals of Danish origin.

For part 1 of the study, we did sensitivity analyses using the CKD-EPI equation to estimate GFR. ${ }^{23}$ We used the tests of the nonzero slope to test for proportional hazard assumptions. The study was approved by the Danish Data Protection Agency (journal no 2008-41-1781). Stata software, Version 11.0 (StataCorp, College Station, TX, USA) and R version 3.1.3 were used to perform statistical analyses.

\section{Results \\ Overall}

On December 31, 2013, a total of 6,239 PLHIV were registered in DHCS. Approximately 1,707 had missing data on smoking, and 17 had aRRT before inclusion (five patients [0.3\%] among never smokers, three patients [0.4\%] among previous smokers, and nine patients [0.4\%] among current smokers) leaving 4,515 PLHIV in the study with a total of 29,196 person years of follow-up (PYFU).

Approximately 1,475 (32.7\%) PLHIV were categorized as never smokers, $768(17.0 \%)$ as previous smokers, and 2,272 $(50.3 \%)$ as current smokers. Median age among previous smokers (39.7 years, interquartile range [IQR]: 32.3-48.3) was higher than among never and current smokers (35.2 years, IQR: 29.5-43.9; 36.4 years, IQR: 30.4-43.7). Number of deaths during study period was greater among current smokers than among never and previous smokers. Characteristics of the study population are summarized in Table 1.

\section{Part I - smoking, renal function, and CKD}

In part 1, 3,142 PLHIV with at least three $\mathrm{CG}-\mathrm{CrCls}$ available were included. No association between smoking
Table I Characteristics of study population

\begin{tabular}{|c|c|c|c|}
\hline & \multicolumn{3}{|c|}{ Smoking status } \\
\hline & Never & Previous & Current \\
\hline Individuals & $\mathrm{I}, 475$ (32.7) & $768(17.0)$ & $2,272(50.3)$ \\
\hline Follow-up (years) & 9,694 & 5,000 & 14,502 \\
\hline Loss to follow-up & $2(0.1)$ & $3(0.4)$ & $5(0.2)$ \\
\hline Emigrated & $81(4.0)$ & $30(3.9)$ & $75(3.3)$ \\
\hline Deaths & $75(5.1)$ & $81(10.5)$ & $4 I I(18.1)$ \\
\hline Age at study & $35.2(29.5-43.9)$ & $39.7(32.3-48.3)$ & $36.5(30.4-43.7)$ \\
\hline \multicolumn{4}{|l|}{ inclusion (years ${ }^{\mathrm{a}}$ ) } \\
\hline Male & $912(61.8)$ & $617(80.6)$ & I,840 (80.9) \\
\hline \multicolumn{4}{|l|}{ Race $^{b}$} \\
\hline Caucasian & $919(62.2)$ & $628(81.9)$ & I,983 (87.3) \\
\hline African & $406(27.5)$ & $82(10.7)$ & $129(5.7)$ \\
\hline Others/unknown & $150(10.2)$ & $58(7.4)$ & $160(7.0)$ \\
\hline \multicolumn{4}{|c|}{ Route of infection ${ }^{\mathrm{b}}$} \\
\hline MSM & $596(40.4)$ & $404(52.6)$ & I,I I 4 (49.0) \\
\hline IDU & $12(0.8)$ & $37(4.7)$ & $316(14.0)$ \\
\hline Heterosexually & $769(52.0)$ & $277(36.1)$ & $736(32.3)$ \\
\hline Others & $98(6.7)$ & $52(6.6)$ & $106(4.7)$ \\
\hline \multicolumn{4}{|l|}{ Comorbidities } \\
\hline Diabetes & $35(2.4)$ & $19(2.5)$ & $56(2.5)$ \\
\hline Hypertension & $98(6.6)$ & $46(6.1)$ & $99(4.4)$ \\
\hline AIDS & $38(2.6)$ & $40(5.2)$ & $107(4.7)$ \\
\hline $\begin{array}{l}\text { CD4 cell count } \\
(\text { cells } / \mu \mathrm{L})^{\mathrm{a}, \mathrm{b}}\end{array}$ & $435(29 I-600)$ & $410(256-600)$ & $445(273-650)$ \\
\hline Weight (kg) ${ }^{a, b}$ & $74.5(65.0-84.0)$ & $75.0(67.0-84.2)$ & $71.2(63.4-80.0)$ \\
\hline Missing & 491 (33.2) & $238(31.0)$ & $644(28.3)$ \\
\hline $\begin{array}{l}\text { CG-CrCl } \\
(\mathrm{mL} / \mathrm{min})^{\mathrm{a}, \mathrm{b}}\end{array}$ & $102(88-120)$ & 101 (87-II9) & $103(87-121)$ \\
\hline $\begin{array}{l}\text { Missing CG-CrCl } \\
\text { at study inclusion }\end{array}$ & 49I (33.2) & $238(31.0)$ & $644(28.3)$ \\
\hline
\end{tabular}

Notes: Data are numbers (\%) unless otherwise specified; ${ }^{a}$ median (interquartile range); bat study inclusion.

Abbreviations: MSM, men who have sex with men; IDU, intravenous drug user; AIDS, acquired immunodeficiency syndrome; $\mathrm{CG}-\mathrm{CrCl}$, estimated creatinine clearance calculated with the Cockcroft-Gault equation.

status and median $\mathrm{CG}-\mathrm{CrCl}$ was observed (Figure 1A). When modeling risk of CKD, we excluded 128 individuals with CKD prior to study inclusion leaving 3,014 PLHIV with a total of 18,131 PYFU. We identified 180 cases of CKD, 47 among never smokers, 43 among previous smokers, and 90 among current smokers. IRs (per 10,000 PYFU) of CKD were 83.2 (95\% confidence interval [CI]: 62.5-110.8) for never smokers, 132.7 (95\% CI: 98.4-178.9) for previous smokers, and 97.4 (95\% CI: 79.2-119.7) for current smokers. In the unadjusted model, previous smoking was associated with increased risk of CKD compared to never smoking (IRR: 1.6, 95\% CI: 1.1-2.4), whereas current smoking was not associated with risk of CKD (IRR: 1.2, 95\% CI: 0.8-1.6). In the adjusted models, this association was not present (IRR: $1.1,95 \%$ CI: $0.7-1.7$ and IRR: 1.3, 95\% CI: 0.9-1.8) for previous smokers and current smokers (Figure 1B and Table 2), with differences in age 

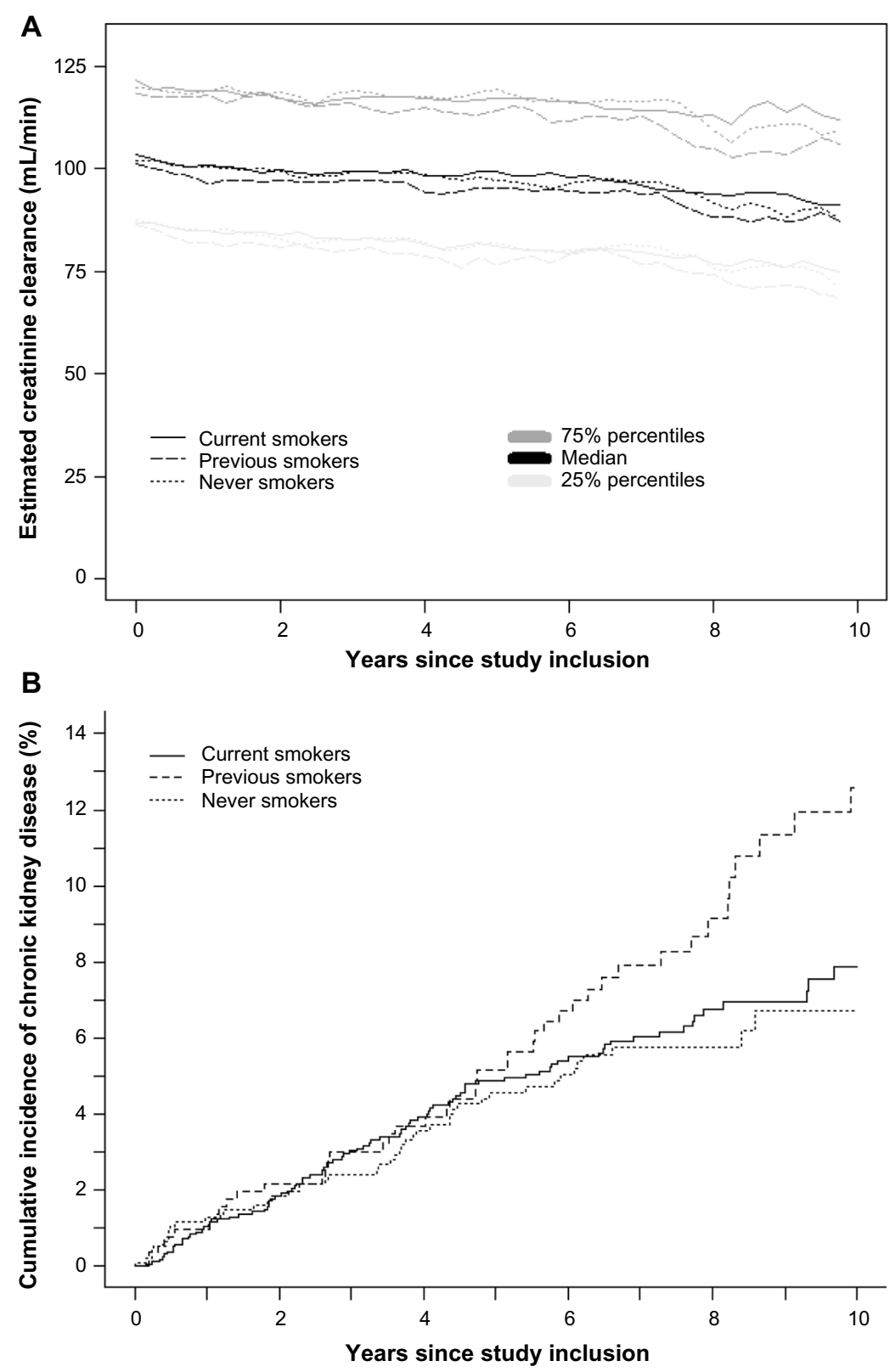

Figure I Estimated creatinine clearance and risk of chronic kidney disease stratified on smoking status.

Notes: (A) 25\%, median, and $75 \%$ percentiles of estimated creatinine clearance stratified on smoking status. We used the Cockcroft-Gault equation to calculate estimated creatinine clearance (displayed as $\mathrm{mL} / \mathrm{min}$ ) as estimate of glomerular filtration rate. We divided time from study inclusion until end of follow-up in 3-month intervals. In each interval, all participants contributed with one CG-CrCl. The CG-CrCl in a time interval was either the median of all CG-CrCls in that time interval. If no CG-CrCl was available in a 3-month time interval, the CG-CrCl was calculated as the weighted mean of the CG-CrCl measurements determined before and after the actual 3-month period. At study inclusion, there were 984 never smokers, 530 previous smokers, and I,628 current smokers. At I0 years of follow-up, II0 never smokers, 98 previous smokers, and 170 current smokers were still under follow-up. (B) Risk of chronic kidney disease stratified on smoking status. Definition of chronic kidney disease: two consecutive CG-CrCls of $\leq 60 \mathrm{~mL} / \mathrm{min} \geq 3$ months apart.

Abbreviation: $\mathrm{CG}-\mathrm{CrCl}$, estimated creatinine clearance calculated with the Cockcroft-Gault equation.

between never smokers and previous smokers explaining most of the risk difference observed between these groups (adjusting for only age - IRR: 1.2, 95\% CI: $0.8-1.8$ and IRR: $1.3,95 \%$ CI: $0.9-1.9$ for previous and current smokers, respectively). As seen in Figure 1B, previous smokers seemed to have increased risk of CKD from 5 years after study inclusion. We therefore performed a Cox regression analysis in which time was split at 5 years after study inclusion. In this analysis, adjusted IRR for previous smokers vs never smokers was 3.4 (95\% CI: 1.3-8.7). When we used the CKD-EPI equation to estimate GFR, it did not change the results substantially (IRR: 1.1, 95\% CI: 0.7-1.6 and IRR: $0.8,95 \% \mathrm{CI}$ : $0.5-1.2$ for previous smokers and current smokers, respectively). 
Table 2 Risk of CKD and aRRT

\begin{tabular}{|c|c|c|c|c|}
\hline \multirow[t]{2}{*}{ Smoking status ${ }^{a}$} & \multirow{2}{*}{$\begin{array}{l}\text { Individuals with CKD } \\
\text { during follow-up }\end{array}$} & \multirow{2}{*}{$\begin{array}{l}\text { IR per } 10,000 \\
\text { PYFU }(95 \% \mathrm{Cl})\end{array}$} & \multicolumn{2}{|l|}{ IRR (95\% CI) } \\
\hline & & & Unadjusted & Adjusted \\
\hline \multicolumn{5}{|l|}{ Risk of CKD } \\
\hline Never & 47 & $83.2(62.5-110.8)$ & Ref & Ref \\
\hline Previous & 43 & | 32.7 (98.4-|78.9) & $1.6(1.1-2.4)$ & I.I (0.7-I.7) \\
\hline Current & 90 & $97.4(79.2-119.7)$ & $1.2(0.8-1.6)$ & I.3 (0.9-I.8) \\
\hline \multicolumn{5}{|l|}{ Risk of aRRT } \\
\hline Never & 20 & $20.6(|3.3-3| .9)$ & Ref & Ref \\
\hline Previous & 11 & $22.0(12.2-39.7)$ & I.I (0.5-2.2) & $0.8(0.4-1.7)$ \\
\hline Current & 31 & $21.4(15.0-30.4)$ & $1.0(0.6-1.8)$ & $0.9(0.5-1.7)$ \\
\hline
\end{tabular}

Notes: at study inclusion; bdefinition of CKD: two consecutive CG-CrCls of $\leq 60 \mathrm{~mL} / \mathrm{min} \geq 3$ months apart. For CKD: adjusted for age, gender, HAART exposure, tenofovir exposure, race, route of infection, comorbidities (diabetes, hypertension, and AIDS), included as time-updated variables, CD4 cell count, and CG-CrCl at study inclusion. For aRRT: adjusted for age, gender, HAART exposure, tenofovir exposure, race, route of infection, comorbidities (diabetes, hypertension, and AIDS), included as time-updated variables and CD4 cell count.

Abbreviations: CKD, chronic kidney disease; aRRT, any renal replacement therapy; IR, incidence rate; PYFU, person years of follow-up; Cl, confidence interval; IRR, incidence rate ratio; CG-CrCl, estimated creatinine clearance calculated with the Cockcroft-Gault equation; HAART, highly active antiretroviral treatment; AIDS, acquired immunodeficiency syndrome; Ref, reference.

\section{Part 2 - smoking and aRRT}

In this part, we included the complete cohort of 4,515 PLHIV with known smoking status. We identified a total of 62 cases of aRRT (20,11, and 31 among never, previous, and current smokers). IRs (per 10,000 PYFU) of aRRT were 20.6 (95\% CI: 13.3-31.9) for never smokers, 22.0 (95\% CI: 12.2-39.7) for previous smokers, and 21.4 (95\% CI: 15.0-30.4) for current smokers. The risk of aRRT in previous and current smokers did not differ substantially from that of never smokers (Figure 2 and Table 2). Excluding IDUs or individuals of non-Danish origin only changed the estimates marginally (results not shown). Only 17 patients (nine never smokers, two previous smokers, and six current smokers) were categorized with cRRT in the study period, and did not allow us to make robust conclusions about association of smoking and risk of cRRT.

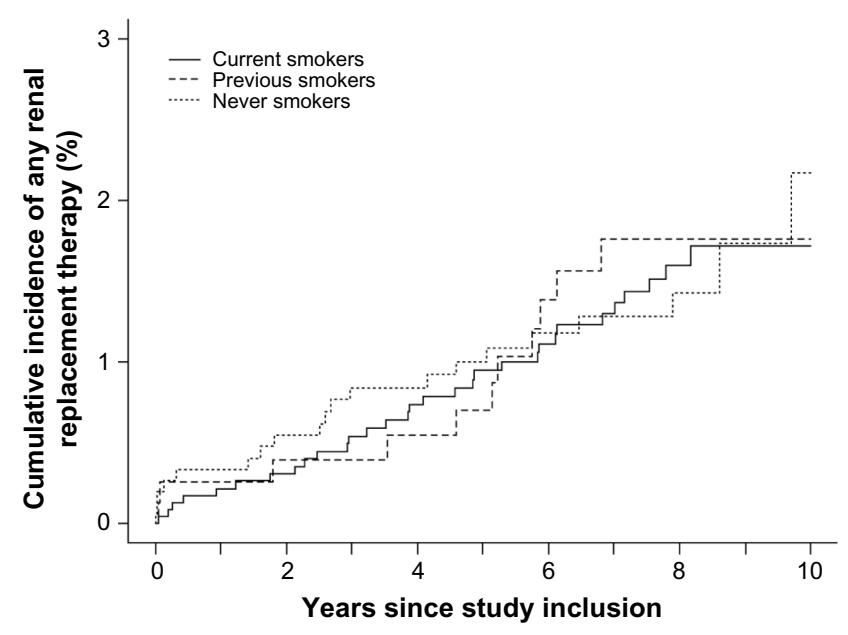

Figure 2 Risk of any renal replacement therapy stratified on smoking status.

\section{Part 3 - smoking and mortality following aRRT}

In this part of the study, we included the 62 individuals from part 2 who initiated aRRT. They had a total of 344 person days of follow-up (PDFU). During the observation time, 26 deaths were recorded, and mortality rates (MRs per 1,000 PDFU) were 29.0 (95\% CI: 10.9-77.3), 65.3 (95\% CI: 23.0-163.0), and 128.0 (95\% CI: 80.6-203.0) for never smokers, previous smokers, and current smokers, respectively. The unadjusted MRRs were 2.0 (95\% CI: 0.5-8.2) and 3.9 (95\% CI: 1.30-11.5) for previous smokers and current smokers, respectively, compared to never smokers (Figure 3). When adjusting for gender and age at study inclusion, the MRRs were 1.9 (95\% CI: 0.5-7.8) and 3.8 (95\% CI: $1.3-11.2)$ for previous and current smokers, respectively, compared to never smokers. Excluding IDUs or individuals

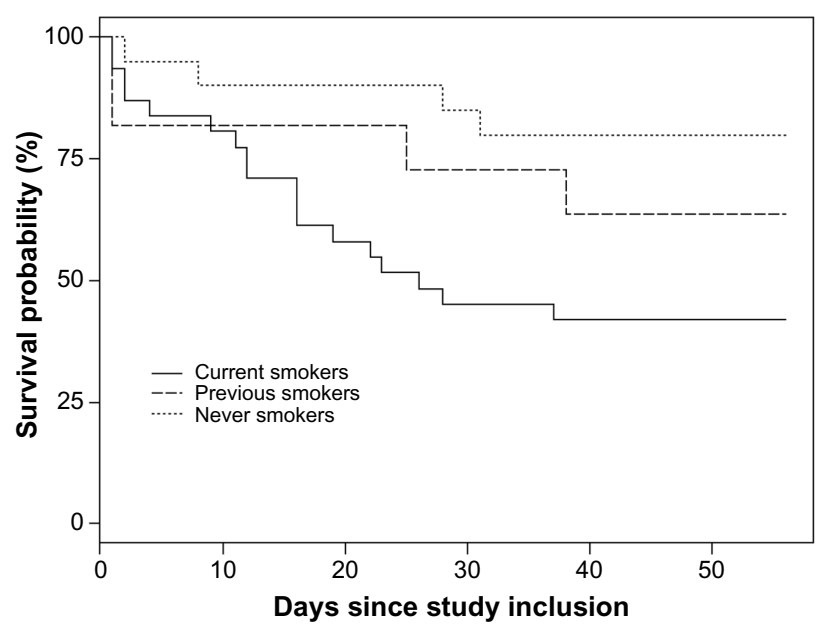

Figure 3 Mortality following any renal replacement therapy stratified on smoking status. 
of non-Danish origin only changed the estimates marginally (results not shown).

\section{Discussion}

In this Danish nationwide population-based cohort study, we found no evidence that smoking status was associated with decline in renal function, risk of CKD, or risk of aRRT. Mortality was high following aRRT, and substantially increased in smokers.

GFR in smokers has not convincingly been shown to be decreased compared to nonsmokers. A large cross-sectional study from France which included 28,409 individuals from the background population found that smoking was associated with a slightly increased CG-CrCl and albuminuria. ${ }^{24}$ Increased GFR and albuminuria in smokers simultaneously have led to the hypothesis that smoking is associated with glomerular hyperfiltration, a phenomenon also described in other disease entities such as diabetes. ${ }^{25,26}$ Thus, glomerular hyperfiltration could be part of the pathogenesis leading to proteinuria in smokers. It could also hide a potential harmful effect of smoking on renal function. Another explanation for an elevated $\mathrm{CG}-\mathrm{CrCl}$ in smokers could be that smokers tend to have more sedentary activities compared to nonsmokers, ${ }^{27}$ which results in lower muscle mass and thereby lower serum creatinine and higher $\mathrm{CG}-\mathrm{CrCl}$. To compensate for differences in muscle mass, we chose to use the CG-CrCl as an estimate of GFR. However, a change in body weight is not solely explained by a change in total muscle mass, and hence, this equation only partly adjusts for differences in muscle mass.

It is controversial whether smoking increases the risk of renal insufficiency in the general population. ${ }^{28}$ Some studies have evaluated the association between smoking and aRRT or ESRD in the general population. Haroun et al found that smoking was associated with ESRD or CKD as cause of death in both men and women with adjusted IRR of 2.4 (95\% CI: 1.4-4.0) and 2.9 (95\% CI: 1.7-5.0) for men and women, respectively. However, this effect was primarily present after the age of $60 .{ }^{29}$ In a study including more than 300,000 individuals from the background population, Klag et al concluded that smoking was significantly associated with ESRD, but the magnitude of the effect of smoking was not reported. ${ }^{30}$ The populations in the above-mentioned studies were older than the population in our study, which may explain some of the discrepancies as only $4 \%$ of the population in our study were older than 60 years of age at study inclusion. We observed a trend toward increased risk of CKD in previous smokers from 5 years after study inclusion. We presume that this is a chance finding but cannot exclude that the morbidity, which prompted smoking cessation, later leads to deteriorating renal function.

Only a few studies have evaluated smoking as a risk factor for renal disease in PLHIV. Ryom et al included smoking as a covariate when evaluating HAART exposure as a risk factor for advanced CKD or ESRD in the D:A:D study. They found that smoking was associated with a statistically significant increased risk of advanced CKD/ESRD (IRR approximately 1.9). ${ }^{31}$ In contrast to our study, the D:A:D study included patients with unknown smoking status at enrollment and was not designed to show effects of smoking on renal function. Furthermore, their population was slightly older than ours. As discussed earlier, one study has indicated that the effect of smoking on the risk of ESRD primarily affects people aged 60 or older, and hence, a potential harmful effect of smoking on renal function may be observed in our population as it grows older. ${ }^{29}$ We have shown that the mortality is higher among smokers than never smokers and previous smokers following aRRT. One could speculate that the reporting of aRRT may be more likely to take place if a patient dies. This could bias the results toward aRRT being associated with smoking. The registration of dialysis in DNHR is coupled to the reimbursement system, and the hospital is only economically compensated if the correct diagnosis is registered in DNHR. We presume that this leads to a high and accurate registration of dialysis, and represents a significant difference from other studies evaluating dialysis as an outcome..$^{32,33}$

Following aRRT, the mortality is increased in smokers compared to never smokers and previous smokers. Increased mortality in smokers while receiving renal replacement therapy is well established. In a large meta-analysis of ESRD patients performed in 2011, Liebman et al found a hazard ratio of 1.65 of death among smokers compared to nonsmokers. ${ }^{34}$ This is comparable to the estimates in our study. However, MRs in the present study are much higher than in previous studies. Our definition of aRRT results in an overrepresentation of patients on acute dialysis, compared to patients on cRRT. Acute dialysis most often occurs with severe acute illnesses such as septic shock with a high mortality, which is the probable explanation for high MRs in our study.

Major strengths of the study are the following: 1) a population-based, nationwide design; 2) the use of DCRS, which assigns a personal identification number to all Danish citizens; 3 ) the well-organized structure of Danish national registers which allowed us to use well-validated data of high quality on vital status; and 4) the use of another well-validated 
Danish database the DNHR which enabled us to extract robust data on study outcomes. ${ }^{20}$

There are some limitations of the study: 1) We did not have access to data on CG-CrCl or aRRT in Danish individuals from the background population with known smoking status. 2) Smoking status was only available for about three quarters of Danish PLHIV. 3) Serum creatinine was measured by different testing methods over the 18-year study period, and we therefore cannot exclude some intra- and interlaboratory variation. ${ }^{35}$ In contrast to previous studies, we corrected for the varying testing methods as suggested by the local laboratories. 4) Our study period started from January 1, 1995, and standardization of serum creatinine measurements was implemented in Denmark around 2004, and hence, a substantial part of the serum creatinine measurements has not been subject to standardization. 5) We chose to use CG-CrCl as an estimate of GFR, since a large number of PLHIV gain weight while recovering from their infection upon commencing HAART. This may introduce a bias as some patients did not have a specific weight available for each serum creatinine measurement. However, a sensitivity analysis using the CKD-EPI equation instead of the Cockcroft-Gault equation gave comparable results. 6) We did not have access to data on proteinuria or albuminuria, and hence, we were not able to test for association between smoking and proteinuria/ albuminuria. 7) Our definition of aRRT included both patients with ESRD on cRRT and patients on acute dialysis. However, IRR of CKD in the present study showed results that only differed marginally from the IRR of aRRT. We therefore believe that our results are rather robust.

We conclude that in Danish PLHIV, the overall renal function, the risk of CKD, or the risk of aRRT is not substantially associated with smoking status, but smoking increases mortality following aRRT.

\section{Acknowledgments}

This work was supported by Preben og Anne Simonsens Foundation, NOVO Nordisk Foundation, Rigshospitalet, Copenhagen University, The Danish AIDS Foundation, and The Augustinus Foundation.

\section{Disclosure}

NO has received research funding from Roche, Bristol-Myers Squibb, Merck Sharp and Dohme, GlaxoSmithKline, Abbott, Boehringer Ingelheim, Janssen-Cilag, and Swedish Orphan. $\mathrm{CP}$ has received research funding from Abbott, Merck Sharp and Dohme, GlaxoSmithKline, and Gilead. JG has received research funding from Abbvie, Bristol-Myers Squibb, Merck Sharp and Dohme, ViiV, Medivir, and Gilead. The other authors report no conflicts of interest. The funding source had no role in the design, conduct, or analysis of the study or the decision to publish the manuscript.

\section{References}

1. Lohse N, Hansen ABE, Pedersen G, et al. Survival of persons with and without HIV infection in Denmark, 1995-2005. Ann Intern Med. 2007; 146(2):87-95.

2. Choi AI, Shlipak MG, Hunt PW, Martin JN, Deeks SG. HIV-infected persons continue to lose kidney function despite successful antiretroviral therapy. AIDS. 2009;23(16):2143-2149.

3. Rasch MG, Engsig FN, Feldt-Rasmussen B, et al. Renal function and incidence of chronic kidney disease in HIV patients: a Danish cohort study. Scand J Infect Dis. 2012;44(9):689-696.

4. Rasch MG, Helleberg M, Feldt-Rasmussen B, et al. Increased risk of dialysis and end-stage renal disease among HIV patients in Denmark compared with the background population. Nephrol Dial Transplant. 2014;29(6):1232-1238

5. Rodriguez-Nóvoa S, Alvarez E, Labarga P, Soriano V. Renal toxicity associated with tenofovir use. Expert Opin Drug Saf. 2010;9(4): 545-559.

6. Eira M, Araujo M, Seguro AC. Urinary NO3 excretion and renal failure in indinavir-treated patients. Braz J Med Biol Res. 2006;39(8): 1065-1070.

7. Daar ES, Tierney C, Fischl MA, et al; AIDS Clinical Trials Group Study A5202 Team. Atazanavir Plus Ritonavir or Efavirenz as Part of a 3-Drug Regimen for Initial Treatment of HIV-1A Randomized Trial. Ann Intern Med. 2011;154(7):445-456.

8. Bruggeman LA, Ross MD, Tanji N, et al. Renal epithelium is a previously unrecognized site of HIV-1 infection. J Am Soc Nephrol. 2000; 11(11):2079-2087.

9. Marras D, Bruggeman LA, Gao F, et al. Replication and compartmentalization of HIV-1 in kidney epithelium of patients with HIV-associated nephropathy. Nat Med. 2002;8(5):522-526.

10. Helleberg M, Afzal S, Kronborg G, et al. Mortality attributable to smoking among HIV-1-infected individuals: a nationwide, populationbased cohort study. Clin Infect Dis. 2013;56(5):727-734.

11. Legarth R, Omland LH, Kronborg G, et al. Employment status in persons with and without HIV infection in Denmark: 1996-2011. AIDS. 2014; 28(10):1489-1498.

12. Helleberg M, Gerstoft J, Afzal S, et al. Risk of cancer among HIVinfected individuals compared to the background population: impact of smoking and HIV. AIDS. 2014;28(10):1499-1508.

13. Rasmussen LD, Helleberg M, May MT, et al. Myocardial infarction among Danish HIV-infected individuals: population attributable fractions associated with smoking. Clin Infect Dis. 2015;60(9):1415-1423.

14. Statistics Denmark [homepage on the Internet]. Population and elections. Available from: http://www.statistikbanken.dk. Accessed January $16,2015$.

15. Lohse N, Obel N, Kronborg G, et al. Declining risk of triple-class antiretroviral drug failure in Danish HIV-infected individuals. Aids. 2005;19:815-822.

16. Helleberg M, Engsig FN, Kronborg G, et al. Retention in a public healthcare system with free access to treatment: a Danish nationwide HIV cohort study. AIDS. 2012;26(6):741-748.

17. Omland LH, Ahlström MG, Obel N. Cohort profile update: the Danish HIV Cohort Study (DHCS). Int J Epidemiol. 2014;0(0):1-6.

18. Obel N, Engsig FN, Rasmussen LD, Larsen MV, Omland LH, Sørensen HT. Cohort profile: the Danish HIV Cohort Study. Int J Epidemiol. 2009;38(5):1202-1206.

19. Schmidt M, Pedersen L, Sørensen HT. The Danish Civil Registration System as a tool in epidemiology. Eur J Epidemiol. 2014;29(8): $541-549$. 
20. Andersen TF, Madsen M, Jørgensen J, Mellemkjær L, Olsen JH. The danish national hospital registry. A valuable source of data for modern health sciences. Dan Med Bull. 1999;46(3):263-268.

21. Cockcroft DW, Gault MH. Prediction of creatinine clearance from serum creatinine. Nephron. 1976;16(1):31-41.

22. Delanaye P, Mariat C, Cavalier E, Krzesinski J-M. Errors induced by indexing glomerular filtration rate for body surface area: reductio ad absurdum. Nephrol Dial Transplant. 2009;24(12):3593-3596.

23. Levey A, Stevens L, Schmid CH, et al. A new equation to estimate glomerular filtration rate. Ann Intern Med. 2009;150(9):604-612.

24. Halimi JM, Giraudeau B, Vol S, et al. Effects of current smoking and smoking discontinuation on renal function and proteinuria in the general population. Kidney Int. 2000;58:1285-1292.

25. Cachat F, Combescure C, Cauderay M, Girardin E, Chehade H. A systematic review of glomerular hyperfiltration assessment and definition in the medical literature. Clin J Am Soc Nephrol. 2015;10(3):382-389.

26. Orth SR. Cigarette smoking: an important renal risk factor - far beyond carcinogenesis. Tob Induc Dis. 2002;1(2):137-155.

27. Strine TW, Okoro CA, Chapman DP, et al. Health-related quality of life and health risk behaviour among smokers. Am J Prev Med. 2005;28(2):182-187.

28. Orth SR, Hallan SI. Smoking: a risk factor for progression of chronic kidney disease and for cardiovascular morbidity and mortality in renal patients - absence of evidence or evidence of absence? Clin J Am Soc Nephrol. 2008;3:226-236.
29. Haroun MK, Jaar BG, Hoffman SC, Comstock GW, Klag MJ, Coresh J. Risk factors for chronic kidney disease: a prospective study of 23,534 men and women in Washington County, Maryland. J Am Soc Nephrol. 2003;14:2934-2941.

30. Klag MJ, Whelton PK, Randall BL, et al. Blood pressure and end-stage renal disease in men. $N$ Engl J Med. 1996;334(1):13-18.

31. Ryom L, Mocroft A, Kirk O, et al. Predictors of advanced chronic kidney disease and end-stage renal disease in HIV-positive persons. AIDS. 2014;28(2):187-199.

32. Nickelsen TN. Datavaliditet of dækningsgrad I Landspatientregistret. En litteraturgennemgang [Data validity and coverage in the Danish National Health Registry. A literature review]. Ugeskr Laeger. 2001; 164(1):33-37. Danish.

33. Blichert-Hansen L, Nielsson MS, Nielsen RB, Christiansen CF, Nørgaard M. Validity of the coding for intensive care admission, mechanical ventilation, and acute dialysis in the Danish national patient registry: a short report. Clin Epidemiol. 2013;5:9-12.

34. Liebman SE, Lamontagne SP, Huang L-S, Messing S, Bushinsky DA. Smoking in dialysis patients: a systematic review and meta-analysis of mortality and cardiovascular morbidity. Am J Kidney Dis. 2011;58(2): 257-265.

35. Miller WG, Myers GL, Ashwood ER, et al. Creatinine measurement: state of the art in accuracy and interlaboratory harmonization. Arch Pathol Lab Med. 2005;129:297-304.
Clinical Epidemiology

\section{Publish your work in this journal}

Clinical Epidemiology is an international, peer-reviewed, open access, online journal focusing on disease and drug epidemiology, identification of risk factors and screening procedures to develop optimal preventative initiatives and programs. Specific topics include: diagnosis, prognosis, treatment, screening, prevention, risk factor modification,

Submit your manuscript here: http://www.dovepress.com/clinical-epidemiology-journal

\section{Dovepress}

systematic reviews, risk \& safety of medical interventions, epidemiology \& biostatistical methods, and evaluation of guidelines, translational medicine, health policies \& economic evaluations. The manuscript management system is completely online and includes a very quick and fair peer-review system, which is all easy to use. 